\title{
OPTIMIZACIÓN DEL RENDIMIENTO DE LA EXTRACCIÓN DE ACEITE DE SEMILLAS DE Vitis vinifera CON CO, SUPERCRÍTICO
}

\author{
Maritza Barriga-Sánchez ${ }^{* a, b}$, Anna Churata Huanca ${ }^{\mathrm{b}}$, Óscar Tinoco Gómez ${ }^{\mathrm{a}}$
}

\begin{abstract}
RESUMEN
Se optimizó el rendimiento de la extracción de aceite de la semilla de uva, variedad Quebranta, con el método superficie respuesta (MSR), se consideraron tres factores: presión (Pbar), temperatura (T) y flujo de $\mathrm{CO}_{2}(\mathrm{~F})$, las variables respuesta fueron el rendimiento de aceite, el valor peróxido (POV) y anisidina. Se caracterizó la semilla y estableció el tamaño de partícula de la semilla para la extracción de aceite. Se realizaron las extracciones según el diseño MSR y se calcularon los rendimientos de aceite, POV y anisidina. Se determinó la composición de ácidos grasos por cromatografía de gases y la actividad antioxidante por reducción del radical 1,1-difenil-2-picrilhidrazil (DPPH) del aceite optimizado obtenido con $\mathrm{CO}_{2}$ y del extraído con hexano (testigo). La Pbar, $\mathrm{T}$ y $\mathrm{F}$ ejercieron efecto significativo sobre el rendimiento, pero no ejercieron efecto sobre el POV y anisidina. Los resultados son prometedores para añadir valor agregado a la semilla, residuo de la elaboración de pisco ya que el aceite contenía $66,45 \%$ de ácido linoléico; $20,05 \%$ de ácido oleico y una capacidad antioxidante de 7,80 $\mathrm{mM}$ equivalente a Trolox.
\end{abstract}

Palabras clave: $\mathrm{CO}_{2}$ supercrítico, pisco, semilla de uva, aceite.

\section{OPTIMIZATION OF SEED OIL EXTRACTION YIELD FROM VITIS VINIFERA WITH SUPERCRITICAL $\mathrm{CO}_{2}$}

\begin{abstract}
The extraction yield of Quebranta grape seed oil was optimized with the response surface method. Three factors were considered: pressure (Pbar), temperature $(\mathrm{T})$ and $\mathrm{CO}_{2}$ flow $(\mathrm{F})$. The response variables were the oil yield, peroxide value (POV) and anisidine. The seed was characterized, and the particle size was established for the oil extraction. The extractions were executed according to the RSM design and the oil yield, POV and anisidine were calculated. The composition of fatty acids was determined by gas chromatography and the antioxidant activity by reduction of the 1,1-diphenyl-2-picrylhydro-radical (DPPH) of

\footnotetext{
${ }^{\text {a }}$ Doctorado de Ciencias Ambientales, Universidad Nacional Mayor de San Marcos, Av. Universitaria /Calle Germán Amézaga 375, Lima 1, Perú, mbarriga@itp.gob.pe

b Dirección de Investigación, Desarrollo, Innovación y Transferencia Tecnológica, Instituto Tecnológico de la Producción
} 
the optimized oil obtained with $\mathrm{CO}_{2}$ and that extracted with hexane (control). The Pbar, T and $\mathrm{F}$ had significant effect on the oil yield, but did not have significant effect on the POV and anisidine. These results were promising to increase added value of the seed and pisco production residue because the oil contained $66.4 \%$ of linoleic acid, $20.05 \%$ of oleic acid and a Trolox equivalent antioxidant capacity of $7.8 \mathrm{mM}$.

Key words: Supercritical $\mathrm{CO}_{2}$, pisco, grape seed, oil.

\section{INTRODUCCIÓN}

El pisco es el aguardiente obtenido exclusivamente por destilación de mostos frescos de "uvas pisqueras" recientemente fermentados, de las zonas de producción reconocidas en el Perú (Norma Técnica Peruana), es una bebida tradicional del Perú.

Los reportes del Instituto Nacional de Estadística muestran producciones de pisco de 2827,8; 3434,5 y 3764,8 miles de litros durante el 2013, 2014 y 2015, respectivamente, generándose grandes cantidades de residuo, conocido como orujo, el cual contiene piel, hollejo y semilla de uva. En Ica lo acumulan fuera de las instalaciones de la planta procesadora de pisco o lo destinan para compostaje, o lo queman causando contaminación ambiental. Considerándose que se requiere de 6 a $7 \mathrm{~kg}$ de uva para producir un litro de pisco ${ }^{1}$ y que el $6 \%$ de la uva corresponde a las semillas, se estima que en el 2015 no se aprovecharon 1581,2 toneladas de semillas, de las que se hubiera extraído aproximadamente $158 \mathrm{t}$ de aceite. El aceite de semilla de uva contiene $70 \%$ de ácido linoléico y $15 \%$ de ácido oleico ${ }^{2}$. El ácido linoléico es un ácido graso esencial, no puede ser sintetizado por los humanos, tiene efectos favorables en colesterol sérico total, tensión arterial procesos inflamatorios y se recomienda su consumo ya que la sustitución de los ácidos grasos saturados por ácidos grasos polinsaturados disminuye el riesgo de enfermedades coronarias ${ }^{3}$.

Además, los compuestos fenólicos de las uvas poseen actividad antioxidante y antimicrobiana y juegan un rol en la prevención de enfermedades cardiovasculares, incluso del cáncer, previene la peroxidación en lípidos del cerebro, del hígado y daños en el DNA en animales ${ }^{4}$. La extracción con $\mathrm{CO}_{2}$ y alcohol de orujo de uva de la variedad uvina, residuo de pisco contiene $23 \pm 0,2$ g equivalente de ácido gálico $/ \mathrm{kg}$ de extracto ${ }^{5}$. Por otro lado, hay una tendencia para mejorar procesos químicos en armonía con el ambiente, como la técnica de extracción con $\mathrm{CO}_{2}$ supercrítico. Su potencia solvente depende esencialmente de la naturaleza del soluto, de la presión y temperatura del sistema ${ }^{2}$; resultando interesante el estudio de la extracción con $\mathrm{CO}_{2}$ supercrítico del aceite de semilla de uva variedad Quebranta de Ica, residuo de la elaboración de pisco, aun no existen estudios con esta tecnología de extracción en el Perú.

El objetivo del estudio fue optimizar el rendimiento de la extracción de aceite de la semilla de uva con $\mathrm{CO}_{2}$ supercrítico y determinar la calidad (POV y anisina) del aceite de cada una de las extracciones. Al aceite extraído con las condiciones óptimas y un aceite extraído con hexano se les determinaron el perfil de ácidos grasos, capacidad antioxidante y otros análisis para evaluar la calidad de aceite. 


\section{PARTE EXPERIMENTAL}

\section{Materiales y métodos}

Se colectaron $60 \mathrm{~kg}$ de orujo (semillas, hollejo y piel) de uva (Vitis vinifera) variedad Quebranta donado por la bodega procesadora de "pisco" Yanquiza, Subtanjalla, Ica (Latitud $14^{\circ} 1$ '17.82"S. Longitud $75^{\circ} 44^{\prime} 35.43^{\prime \prime O}$ ), se mantuvo la cadena de frío hasta su llegada a Lima al laboratorio de fisicoquímica del Instituto Tecnológico de la Producción (ITP), donde el orujo fue colocado en las bandejas del secador de aire frío (Asahi, Ohiya Nishi$\mathrm{Ku}$, Japón) a $25^{\circ} \mathrm{C}$ durante 36 horas hasta $13 \%$ de humedad, el hollejo y piel de uva fueron separados con el tamiz de acero inoxidable (KM Testing sieve, Japón) de $7 \mathrm{~mm}$ de abertura. Las semillas fueron sometidas a secado a $40{ }^{\circ} \mathrm{C}$ por 6 horas en una estufa de convección forzada (Venticell, USA) hasta una humedad menor a $7 \%$. Finalmente, las semillas fueron envasadas al vacío en bolsas de polietileno y guardadas en refrigeración $\left(5 \pm 1{ }^{\circ} \mathrm{C}\right)$ hasta su posterior uso.

Los contenidos de proteínas (factor 6,25), humedad, grasa (éter dietílico) y cenizas de la semilla de uva, fueron determinados por duplicado según los métodos establecidos por la Asociación de Químicos Analíticos Oficiales (AOAC).

\section{Tamaño de partícula de la semilla de uva en la extracción con $\mathrm{CO}_{2}$ supercrítico}

Con la finalidad de mejorar el rendimiento, se trituró la semilla con un molino (Ika A11, USA), se separaron dos grupos, el primero que pasó el tamiz de $1 \mathrm{~mm}$ (Retsch, Alemania) y se quedó retenido en el tamiz de 0,85 mm y el segundo grupo pasó el tamiz de 0,71 mm (Mesh 25). Se realizaron extracciones de aceite por triplicado de cada grupo a 300 bar, 45 ${ }^{\circ} \mathrm{C}$ y flujo de $70 \mathrm{~g} / \mathrm{min}$, (condiciones recomendadas por el fabricante del equipo), durante 2 horas. Se determinó el rendimiento $(\mathrm{R})$ usando la ecuación 1

$$
R=\frac{m \text { aceite }}{m s s} x 100
$$

Donde:

$\mathrm{R}=\%$ en $\mathrm{g}$ de aceite $/ 100 \mathrm{~g}$ semilla seca

$m_{\text {aceite }}=$ Peso del aceite $(\mathrm{g})$.

$m_{s s}=$ Peso de la semilla seca molida sometida a extracción.

\section{Análisis estadísticos}

Se usó el software estadístico Minitab® (versión 17, USA).

Se compararon las medias de los grupos de semillas con la prueba $\mathrm{T}$ de student y nivel de confianza de $95 \%$.

La optimización del rendimiento de aceite con $\mathrm{CO}_{2}$ supercrítico fue realizado con metodología superficie respuesta (MSR) se consideraron tres factores: presión, temperatura y flujo, los niveles superiores e inferiores de los factores (tabla 1) fueron establecidos según resultados de las pruebas preliminares, los parámetros supercríticos del $\mathrm{CO}_{2} \mathrm{y}$ el funcionamiento del extractor supercrítico. 
En el diseño compuesto central (DCC) cada factor tenía 5 niveles (tabla 1) y 3 réplicas en el punto central, con un total de 17 experimentos.

Tabla 1. Niveles de los factores del diseño compuesto central.

\begin{tabular}{lccccc}
\hline Variable & & \multicolumn{4}{c}{ Niveles } \\
& $-\boldsymbol{\alpha}=-\mathbf{1 , 6 8}$ & $\mathbf{- 1}$ & $\mathbf{0}$ & $\mathbf{1}$ & $\boldsymbol{\alpha}=\mathbf{1 , 6 8}$ \\
\hline Presión $(\mathrm{P})$ bar & 142,7 & 170 & 210 & 250 & 277,3 \\
Temperatura $(\mathrm{T})^{\circ} \mathrm{C}$ & 33,2 & 40 & 50 & 60 & 66,8 \\
Flujo $(\mathrm{F}) \mathrm{g} / \mathrm{min}$ & 23,2 & 30 & 40 & 50 & 56,8 \\
\hline
\end{tabular}

\section{Extracción del aceite}

Con $\mathrm{CO}_{2}$ supercrítico: en el vaso de extracción de $29 \mathrm{~mm}$ de diámetro y $147 \mathrm{~mm}$ de longitud, se añadió $35 \pm 1 \mathrm{~g}$ de semilla seca y molida $\left(\mathrm{m}_{\mathrm{ss}}\right)$, previamente pasada por el tamiz de mesh 25. El vaso fue colocado en el reactor del equipo extractor multisolvente 2802.0000 (Top industrie, Francia). En el software se programó la temperatura del pre-calentador, reactor y separadores y el flujo del $\mathrm{CO}_{2}$, para llegar a la presión se usó una válvula en forma manual. Luego de llegar a las condiciones de temperatura y flujo y presión del diseño DCC (área sombreada de la tabla 2), se inició la extracción durante dos horas. Se determinó R según la ecuación 1.

Con hexano: se pesaron 5 a 6 g de semilla seca y molida y en un dedal fueron colocados en la cámara del sistema soxhlet, con $120 \mathrm{ml}$ de hexano (99,5\% v/v, JT.Baker, México). La extracción duró seis horas. Se determinó R según la ecuación 1.

\section{Determinaciones analíticas}

Los ácidos grasos metilados del aceite optimizado y al extraído con hexano fueron cuantificados con el software del cromatógrafo de gases (Perkin Elmer Autosystem XL, USA) con detector tipo FID, columna Supelcowax-10 (Merck, Alemania) de $30 \mathrm{~m}$ de longitud, $0,25 \mathrm{~mm}$ de diámetro interno y $0,25 \mu \mathrm{m}$ de espesor de película. Las condiciones de análisis fueron: horno: $160-230{ }^{\circ} \mathrm{C}$, inyector: $250{ }^{\circ} \mathrm{C}$; detector: $270{ }^{\circ} \mathrm{C}$; la presión del hidrógeno 5 psi; split 100:1; volumen de inyección $2 \mu 1$. La identificación de los ácidos grasos se realizó con esteres metílicos de ácidos grasos FAME's (Restek, Bellafonte. USA). Se reportaron los resultados como gramos de ácido graso/100 g de aceite.

El valor peróxido (POV), ácidos grasos libres y el índice de yodo fueron determinados según la AOAC y el valor de anisidina según la norma técnica peruana con el espectrómetro UV visible Perkin Elmer (Lambda 950, USA).

La capacidad antioxidante se realizó por reducción del radical 1,1-difenil-2-picrilhidrazil (DPPH) (Alfa Aesar, España), se realizaron las lecturas a $515 \mathrm{~nm}$ con el espectrómetro UV visible Perkin Elmer (Lambda 950, USA), se expresó en mM equivalente trolox. 


\section{RESULTADOS Y DISCUSIÓN}

Las semillas de uva variedad quebranta contenían $6,49 \pm 0,09 \%$ de agua, $12,47 \pm 0,32 \%$ de grasa cruda (extracción con éter dietílico). Los valores de grasa fueron superiores a los encontrados por Özcan et al. ${ }^{6} \mathrm{y}$ similares a los encontrados por otros autores ${ }^{7}$.

\section{Tamaño de partícula en la extracción supercrítica}

Los contenidos de aceite extraídos con $\mathrm{CO}_{2}$ supercrítico de la semilla de 0,85-1mm eran inferiores que el contenido de grasa cruda, los bajos rendimientos se deben a que el aceite quedó retenido en el interior de las partículas. Se obtuvieron mayores rendimientos de aceite con tamaños de partículas $<0,71 \mathrm{~mm}$ (Fig. 1), debido a que existe mayor superficie de contacto con $\mathrm{CO}_{2}$ supercrítico facilitando la mayor solubilización y extracción del aceite de la semilla. Este resultado coincide con Fiori et $a l^{8}$, quienes obtuvieron mayores rendimientos con partículas de 0,39 a $0,5 \mathrm{~mm}$.

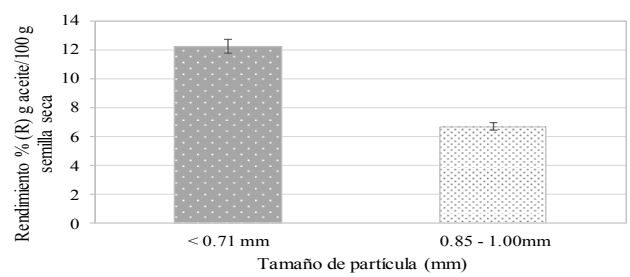

Figura 1. Rendimiento de aceite con $\mathrm{CO}_{2}$ supercrítico de las semillas de uva con diferentes tamaños de partícula.

\section{Análisis del DCC de optimización de la extracción de aceite con $\mathrm{CO}_{2}$ supercrítico}

La presión (Pbar), temperatura $(\mathrm{T})$, flujo $(\mathrm{F})$ y la interacción entre ellos ejercieron un efecto significativo $(\mathrm{P}<0,05)$ sobre el rendimiento de aceite (tabla 2$)$. El coeficiente de determinación del modelo fue de $96,73 \%$, indicando que el modelo predictivo fue altamente significativo. El análisis de regresión de superficie respuesta reporta el modelo predictivo de optimización

$\mathrm{R}=-23,4+0,1973 \mathrm{P}-0,614 \mathrm{~T}+1,134 \mathrm{~F}+0,000444 \mathrm{P} 2+0,003559 \mathrm{PxT}-0,002690 \mathrm{PxF}-$ $0,00838 \mathrm{TxF}$

Por otro lado, la presión, temperatura, flujo no presentaron un efecto significativo sobre los valores de POV y anisidina del aceite extraído con $\mathrm{CO}_{2}$ supercrítico (valores de ANOVA no mostrados $(\mathrm{P}>0,05)$. 
Tabla 2. Diseño compuesto central (DCC) y variables respuesta.

\begin{tabular}{|c|c|c|c|c|c|c|}
\hline \multirow[b]{2}{*}{ Experimentos } & \multicolumn{3}{|c|}{ Variable independiente } & \multicolumn{3}{|c|}{ Variable dependiente } \\
\hline & $\begin{array}{l}\text { Presión } \\
\text { (bar) }\end{array}$ & $\begin{array}{l}\text { Temperatura } \\
\left({ }^{\circ} \mathrm{C}\right)\end{array}$ & $\begin{array}{l}\begin{array}{l}\text { Flujo } \\
(\mathrm{g} / \mathrm{min})\end{array} \\
\end{array}$ & $\begin{array}{l}\mathrm{Rg} \\
\text { aceite/100g }\end{array}$ & $\begin{array}{l}\mathrm{POV} \\
\text { mequivO }_{2} / \mathrm{kg}\end{array}$ & Anisidina \\
\hline 1 & 170 & 40 & 30 & 7,18 & 0,77 & 5,32 \\
\hline 2 & 250 & 40 & 30 & 13,75 & 2,06 & 3,17 \\
\hline 3 & 170 & 60 & 30 & 2,37 & 1,88 & 4,46 \\
\hline 4 & 250 & 60 & 30 & 12,95 & 1,27 & 4,34 \\
\hline 5 & 170 & 40 & 50 & 14,19 & 1,12 & 4,20 \\
\hline 6 & 250 & 40 & 50 & 14,77 & 0,48 & 3,05 \\
\hline 7 & 170 & 60 & 50 & 4,34 & 0,71 & 4,32 \\
\hline 8 & 250 & 60 & 50 & 12,30 & 0,18 & 3,30 \\
\hline 9 & 142,7 & 50 & 40 & 3,69 & 0,54 & 3,70 \\
\hline 10 & 277,3 & 50 & 40 & 14,76 & 0,68 & 3,30 \\
\hline 11 & 210 & 33,2 & 40 & 13,20 & 2,30 & 2,94 \\
\hline 12 & 210 & 66,8 & 40 & 7,49 & 2,30 & 3,20 \\
\hline 13 & 210 & 50 & 23,2 & 7,37 & 1,19 & 4,01 \\
\hline 14 & 210 & 50 & 56,8 & 13,95 & 1,90 & 2,34 \\
\hline 15 & 210 & 50 & 40 & 11,70 & 2,34 & 2,16 \\
\hline 16 & 210 & 50 & 40 & 12,37 & 1,34 & 2,31 \\
\hline 17 & 210 & 50 & 40 & 12,01 & 1,70 & 2,25 \\
\hline
\end{tabular}

Aizpurua et al. ${ }^{9}$ mencionaron que la temperatura y tiempo no ejercieron efecto en el rendimiento de aceite en semilla de uva pero que el flujo y presión sí fueron significativos, caso contrario se demostró en este trabajo la temperatura sí ejerció efecto sobre el rendimiento de aceite, sin embargo, la temperatura óptima de Aizpurua et al. ${ }^{9}$ fue muy cercana a la que se encontró en este trabajo.

Tabla 3. ANOVA de la regresión de superficie respuesta $P, T$ y $F$ versus el rendimiento (R) ( $\mathrm{g}$ aceite/100 $\mathrm{g}$ de semilla de uva deshidratada).

\begin{tabular}{lrrrrr} 
Fuente & GL & $\begin{array}{c}\text { Suma } \\
\text { cuadrados }\end{array}$ & $\begin{array}{c}\text { Suma } \\
\text { medias }\end{array}$ & \multicolumn{1}{c}{ Falor } & \multicolumn{1}{c}{ Valor } \\
\hline Modelo & 9 & 271,542 & 30,171 & 42,59 & 0 \\
Lineal & 3 & 229,762 & 76,587 & 108,11 & 0 \\
Presión & 1 & 143,655 & 143,655 & 202,77 & 0 \\
Temperatura & 1 & 55,552 & 55,552 & 78,41 & 0 \\
Flujo & 1 & 30,556 & 30,556 & 43,13 & 0 \\
Cuadrático & 3 & 10,679 & 3,56 & 5,02 & 0,036 \\
PxP & 1 & 9,737 & 9,737 & 13,74 & 0,008 \\
TxT & 1 & 3,231 & 3,231 & 4,56 & 0,07 \\
FxF & 1 & 2,012 & 2,012 & 2,84 & 0,136 \\
Interacción & 3 & 31,1 & 10,367 & 14,63 & 0,002 \\
PxT & 1 & 16,216 & 16,216 & 22,89 & 0,002 \\
PxF & 1 & 9,265 & 9,265 & 13,08 & 0,009 \\
TXF & 1 & 5,619 & 5,619 & 7,93 & 0,026 \\
Error & 7 & 4,959 & 0,708 & & \\
Lack-of-Fit & 5 & 4,737 & 0,947 & 8,54 & 0,108 \\
Error & 2 & 0,222 & 0,111 & & \\
Total & 16 & 276,501 & & & \\
\hline
\end{tabular}

GL: grados de libertad 
A mayores temperaturas $\left(>60^{\circ} \mathrm{C}\right)$ el $\mathrm{R}$ de extracción de aceite es minimo o nulo, inclusive a mayores flujos no se mejora el rendimiento, pero a temperaturas menores y mayor flujo de $\mathrm{CO}_{2}$ se obtienen mayores $\mathrm{R}$ (figuras $2 \mathrm{a}$ y $3 \mathrm{a}$ ). Otra manera de lograr elevados rendimientos es a altas presiones y mayores flujos (figuras $2 \mathrm{~b}$ y $3 \mathrm{~b}$ ), a baja temperatura y en el rango de las presiones en estudio también se obtiene mayor $\mathrm{R}$, pero a alta temperatura se requiere mayor presión (Figs. 2c y 3c), esas condiciones de temperatura y presión permiten aumentar la densidad de $\mathrm{CO}_{2}$. Se requiere densidades de $\mathrm{CO}_{2}$ de $0,8 \mathrm{~g} / \mathrm{ml}$ o mayores para la extracción de lípidos y para lograr esa densidad se debe elevar la presión a medida que la temperatura sea mayor ${ }^{10}$.
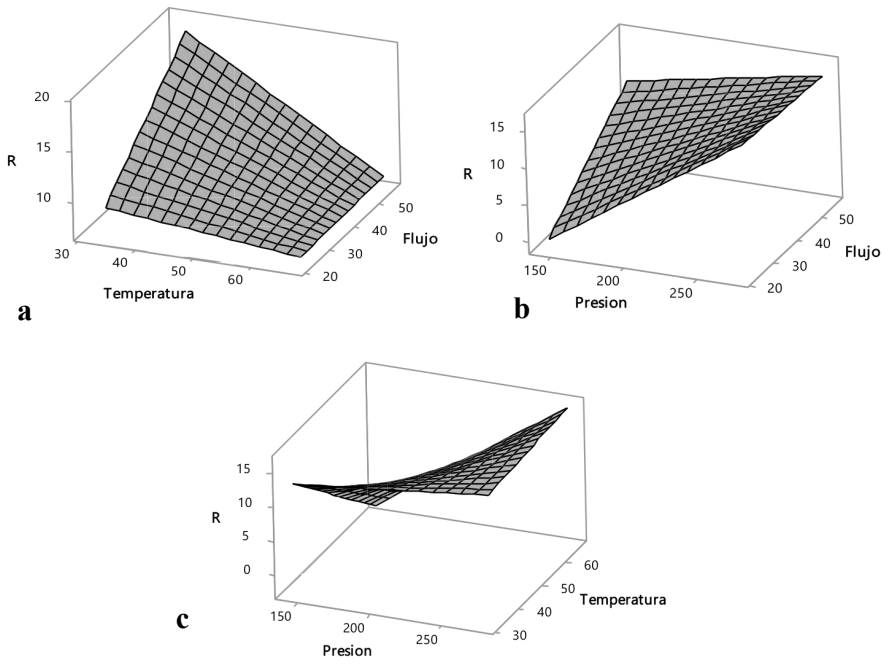

Figura 2. Diagrama superficie respuesta a) Efecto de la $\mathrm{T}$ y $\mathrm{F}$ a 210 bar. b) Efecto de $\mathrm{P}$ y $\mathrm{F}$ a $50{ }^{\circ} \mathrm{C}$. c) Efecto de a $\mathrm{P}$ y $\mathrm{T}$ a $40 \mathrm{~g} \mathrm{CO} 2 / \mathrm{min}$
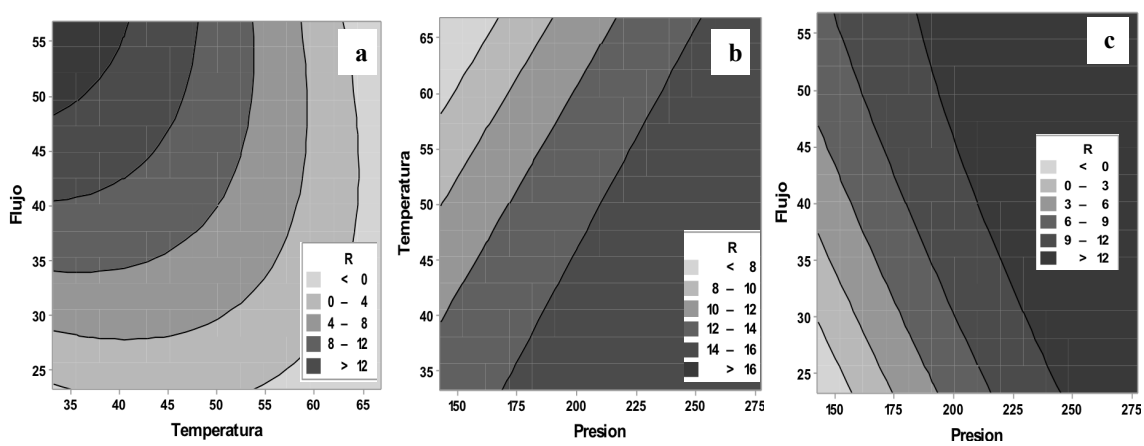

Figura 3. Diagrama de contorno de rendimiento de aceite a) Efecto del flujo y temperatura a 210 bar. b) Efecto de la temperatura y presión a $40 \mathrm{~g} \mathrm{CO} 2 / \mathrm{min}$ c) Efecto del flujo y presión a $50{ }^{\circ} \mathrm{C}$ 
Los parámetros optimizados del rendimiento de aceite fueron 188 bar, $33,5^{\circ} \mathrm{C}$ y $57 \mathrm{~g} / \mathrm{min}$, con esa condición se obtuvo un rendimiento de $16,5 \pm 0,4 \%$, superior al $\mathrm{R}$ obtenido con hexano $(14,45 \pm 0,29 \%)$, a la grasa cruda de la semilla obtenido con éter dietilico $(12,47 \pm$ $0,4 \%)$ y a los encontrados por Passos et al. ${ }^{11}$.

\section{Perfil de ácidos grasos}

Los ácidos grasos encontrados de mayor a menor porcentaje fueron: linoléico (18:2), oléico (18:1), palmítico (16:0), esteárico (18:0), y en pequeñas cantidades el linolénico (18:3) (tabla 4 ), estuvieron en el rango encontrado por otros autores ${ }^{6}$ y dentro de los rangos establecidos por el Codex Stan 210-1999. El resultado es prometedor para la valorización de la semilla de uva del residuo de elaboración de pisco, los ácidos grasos linoléico y oleico son de alto valor nutricional ${ }^{9}$

Tabla 4. Perfil de ácidos grasos del aceite de semilla de uva en g ácidos graso/100 g aceite.

\begin{tabular}{lll}
\hline Ácido graso & $\mathbf{C O}_{2}$ & Hexano \\
\hline C 16:0 (Palmítico) & $6,71 \pm 0,2$ & $7,52 \pm 0,4$ \\
C 18:0 (Esteárico) & $4,81 \pm 0,3$ & $4,44 \pm 0,5$ \\
C 18:1 w-9 (Oleico) & $20,17 \pm 0,3$ & $19,65 \pm 0,4$ \\
C 18:2 w-6 (Linoléico) & $66,69 \pm 0,9$ & $66,69 \pm 0,4$ \\
C 18:3 w-3 (a-Linolénico) & $0,31 \pm 0,1$ & $0,30 \pm 0,1$ \\
C 20:0 (Araquídico) & $0,21 \pm 0,1$ & $0,16 \pm 0,1$ \\
\hline
\end{tabular}

\section{Valores de POV, anisidina, acidez libre e índice de yodo del aceite optimizado extraído con CO2 supercrítico en comparación con el aceite extraído con hexano}

Los valores de POV del aceite extraído con $\mathrm{CO}_{2}$ estuvieron debajo del límite (15 miliequivalentes de $\mathrm{O}_{2} / \mathrm{kg}$ aceite) establecido por el Codex Stan 210-1999, incluso el aceite extraído a la mayor temperatura $\left(66,8^{\circ} \mathrm{C}\right)$, debido a que en esta técnica de extracción el $\mathrm{CO}_{2}$ solubiliza el aceite de la semilla, se separa el $\mathrm{CO}_{2}$ con el aceite y pasa a un separador con menor temperatura, y el aceite es colectado, en este caso, el aceite no tiene una exposición prolongada al calor en comparación con la técnica de extracción con hexano. En la tabla 5 se observa que el aceite extraído con hexano superó el límite establecido por el Codex Stan 210-1999, similar resultado al obtenido por Franco-Mora et al. ${ }^{12}$, los altos valores de POV es una consecuencia del proceso de extracción con solvente que favorece la descomposición de las cadenas de triglicéridos debido a la exposición prolongada a alta temperatura, acelerando el proceso de oxidación y afectando la calidad del aceite ${ }^{12}$. La técnica de extracción de aceite influye en el valor peróxido, el aceite obtenido mediante prensa mecánica presenta valores bajos de $\mathrm{POV}^{13}$.

En este trabajo los resultados de los valores de acidez y anisidina de ambas extracciones (tabla 5) fueron menores que el límite establecido por el Codex Stan 210-1999 y Codex on Fats and Oils ${ }^{14}$. 
En relación al índice de yodo, el aceite extraído con $\mathrm{CO}_{2}$ presentó valor cercano al rango inferior establecido por el Codex Stan 210-1999, Franco-Mora et al. ${ }^{12}$ encontraron en la semilla de uva el valor de índice de yodo de $57,9 \mathrm{~g} / 100 \mathrm{~g}$, mencionaron que los bajos valores corresponden a un aceite altamente poliinsaturado, en ese caso el aceite contenía $90 \%$ de ácidos grasos insaturados.

Tabla 5. Características químicas del aceite de semilla de uva Quebranta extraído con $\mathrm{CO}_{2}$ supercrítico y por el método convencional con el equipo soxhlet.

\begin{tabular}{llllc}
\hline $\begin{array}{l}\text { Aceite de semillas de } \\
\text { uva Quebranta }\end{array}$ & $\begin{array}{l}\text { Índice de peróxido } \\
(\mathbf{m E q} / \mathbf{k g})\end{array}$ & $\begin{array}{l}\text { Índice de } \\
\text { anisidina }\end{array}$ & \multicolumn{2}{c}{$\begin{array}{c}\text { Acidez libre (g } \\
\text { oleico/100g aceite) }\end{array}$} \\
\hline $\begin{array}{l}\text { Extracción convencional de yodo } \\
\text { (g/100g) }\end{array}$ \\
\hline $\begin{array}{l}\text { (hexano) } \\
\text { Extracción con } \mathrm{CO}_{2}\end{array}$ & $38,44 \pm 0,44$ & $3,06 \pm 0,15$ & $0,82 \pm 0,04$ & $130,48 \pm 0,06$ \\
\hline
\end{tabular}

\section{La capacidad antioxidante}

La capacidad antioxidante del aceite extraído con $\mathrm{CO}_{2}$ supercrítico $(7,80 \mathrm{mM}$ equivalente Trolox) y con hexano (7,26 mM equivalente a Trolox) de la uva variedad quebranta del residuo del procesamiento de pisco, presentaron diferencia, posiblemente por efecto de la temperatura el aceite extraído con hexano presentó menor capacidad antioxidante (aproximadamente $7 \%$ menos), por otro lado, Gómez y Huacayo ${ }^{15}$ determinaron una mayor capacidad antioxidante en el aceite de semillas de uva variedad quebranta de Majes (Arequipa) extraído por prensado en frío (9,50 mM equivalente a Trolox), si bien es cierto la variedad de uva en estudio es la misma, esta diferencia puede deberse a la diferencia en la técnica de extracción o a la diferente zona de cultivo de la uva y/o que la semilla del presente trabajo es residuo del procesamiento de pisco, además, es importante mencionar que Gómez y Huacayo ${ }^{15}$ lograron un menor rendimiento del aceite $(7,2 \%)$ que equivale al $44 \%$ encontrado en el presente trabajo $(16,5 \%)$.

\section{CONCLUSIONES}

- $\quad$ El análisis del diseño central compuesto permitió demostrar el efecto significativo de la temperatura, presión y flujo de $\mathrm{CO}_{2}$, así como la interacción de ellos sobre el rendimiento de aceite.

- Las condiciones óptimas de extracción de aceite de semilla de uva con $\mathrm{CO}_{2}$ supercrítico, sugeridas por el método superficie respuesta, fueron 188 bar, $33,5{ }^{\circ} \mathrm{C}$ y $57 \mathrm{~g} / \mathrm{min}$, logrando mejorar el rendimiento de extracción de aceite.

- Los contenidos de ácido linoléico y ácido oleico, así como la actividad antioxidante del aceite de semilla de uva variedad Quebranta, son resultados prometedores para la valorización de la semilla, residuo de la elaboración de pisco. 


\section{AGRADECIMIENTO}

Los autores agradecen a InnovatePerú por el financiamiento de la compra del equipo extractor Multisolvente Proyecto 315- PNICP -EC-2014, así mismo se agradece al Ing. Jorge Torres de la Cite Agroindustrial Ica por la recolección y envío de la muestra de orujo. Al Instituto Tecnológico de la Producción por brindar las facilidades de uso de los equipos e instalaciones.

\section{REFERENCIAS BIBLIOGRÁFICAS}

1. Programa de las Naciones Unidas (PNUD) y Centro de Innovación Tecnológica vitivinícola (CITEVID). La uva y el pisco. Potencialidades productivas. [Internet] 2004. [consultado 05 set 2017]. Disponible en: http://cendoc.esan.edu.pe/fulltext/edocuments/PNUD/Pub_UvaPisco.pdf

2. Duba KS, Fiori L. Supercritical CO2 extraction of grape seed oil: Effect of process parameters on the extraction kinetics. J Supercrit Fluids. 2015; 98: 33-43.

3. Organización de las Naciones unidas para la Alimentación y Agricultura (FAO) y la Fundación Iberoamericana de Nutrición (FINUT). Grasas y ácidos grasos en nutrición humana Consulta de expertos 91. Granada, España; FAO. 2012

4. Bagchi D, Bagchi M, Stohs SJ, Das DK, Ray, SD, Kuszynski CA. et al. Free radicals and grape seed proanthocyanidin extract: importance in human health and disease prevention. Rev Toxicol. 2000; 148 (2-3): 187-197.

5. Farías-Campomanes AM, Rostagno MA, Meireles AA. Production of polyphenol extracts from grape bagasse using supercritical fluids: Yield, extract composition and economic evaluation. J Supercrit Fluids. 2013; 77: 70-78.

6. Özcan, M, Ünver A, Gümüş T. Akın A. Characteristics of grape seed and oil from nine Turkish cultivars. Nat Prod Res. 2012; 26: 2024-2029.

7. Wen Xin, Zhu Minghui, Hu Rui, Zhao Jinhong, Chen Ziye, Li Jingming. et al. Characterization of seed oils from different grape cultivars grown in China. J Food Sci Technol. 2016, 53(7), 3129-3136.

8. Fiori L. Grape seed oil supercritical extraction kinetic and solubility data: Critical approach and modeling. J Supercrit Fluids. 2007; 43: 43-54.

9. Aizpurua-Olaizola O, Ormazábal M, Vallejo A, Olivares M, Navarro P, Etebarria N, et al. Optimization of supercritical Fluid Consecutive Extraction of Fatty Acids and Polyphenols from Vitis vinifera Grape Wastes. J Food Sci. 2015; 80: 101-107.

10. Cunico LP, Turner C. Supercritical Fluids and Gas-Expanded Liquids. En F. Pena-Pereira y M. Tobiszewski (Eds.), The Application of Green Solvents in Separation Processes. Atlanta:Elsevier; 2017. p 155-214.

11. Passos C, Silva, R, Da Silva F, Coimbra M, Silva C. Enhancement of the supercritical fluid extraction of grape seed oil by using enzymatically pre-treated seed. J Supercrit Fluids. 2009; 48: 225-229.

12. Franco-Mora, O, Salomon-Castaño J, Morales AA, Castañeda-Vildózola A, RubíArriaga M. Ácidos grasos y parámetros de calidad del aceite de semilla de uva silvestre (Vitis spp.). Scientia Agropecuaria. 2015; 6(4): 271-278. 
13. Navas PB. Composición química del aceite virgen obtenido por extracción mecánica de algunas variedades (Vitis vinifera L.) con énfasis en los components minoritarios. Arch Latinoam Nutr. 2009; 59(2): 214-219.

14. CODEX. Report of The 25th Session Of The Codex Committee On Fats And Oils. 27 February - 03 March 2017; Kuala Lumpur, Malaysia.

15. Gómez A, Huacayo LM. Efecto del aceite de semilla de Vitis vinífera L. (Uva) sobre la hipertensión arterial experimentalmente inducida en ratas. [Tesis de grado]. Arequipa, Perú: Universidad Católica de Santa María; 2014. 